\title{
Nanovaccine Development for Cocaine Addiction: Immune Response and Brain Behaviour
}

\author{
Rajagopal Appavu* \\ University of Texas Medical Branch, 301 University Blvd, Galveston, TX 77555, USA
}

"Corresponding author: Rajagopal Appavu, Department of Pharmacology and Toxicology, Sealy Center for Vaccine Development, Basic Sciences Building 3.306, University of Texas Medical Branch, 301 University Blvd, Galveston, TX, USA, Tel: 409-256-9987; E-mail: dominiquerajagopal@gmail.com; raappavu@utmb.edu

Received date: February 29, 2016; Accepted date: March 25, 2015; Published date: March 30, 2015

Copyright: @ 2016 Appavu R, This is an open-access article distributed under the terms of the Creative Commons Attribution License, which permits unrestricted use, distribution, and reproduction in any medium, provided the original author and source are credited.

\begin{abstract}
Currently, there are no vaccines to fight against cocaine addiction for use in humans. Clinical trials for a few competitor antibodies are in different stages and a protected and viable immunization is sought after before the end of 2016 .
\end{abstract}

\section{Introduction}

The biology of cocaine addiction is complex but can be interrupted using cocaine vaccines. After cocaine intake, cocaine molecules are captured in the blood circulation system, cross the blood-brain barrier, and move into the cerebrum causing dopamine levels to spike and creating subjective impacts including hyperactivity and intense pleasure. The thought behind cocaine immunization is to get into the body's existing immunologic framework to generate antibodies which can bind to cocaine in the bloodstream and prevent it from reaching the brain. Because of a specific binding capacity toward cocaine molecules, these antibodies can maintain cocaine from entering into the brain and successfully repress its pleasurable impacts.

\section{Immune response and anti-cocaine vaccine}

To become immune to cocaine addiction, the body must be persuaded to mount an antibody response against cocaine molecules when they enter the circulatory system. Since cocaine is a very small atom, it successfully evades the natural antibody response and is said to be "non-antigenic". In an effort to address this problem, researchers have successfully conjugated cocaine molecules to a non-lethal, highly antigenic cholera poison (subunit B) - which can be a nanoprotein/ naopeptide [1,2] including nano-aromatic proteins/peptide emitted from the cholera microscopic organisms. This hybrid cocaine/cholera subunit $\mathrm{B}$ acts much like existing conjugate vaccines used to prevent Haemophilus influenza and Streptococcus pneumoniae. Once inoculated into the body, this hybrid cocaine/cholera subunit B protein induces a potent antibody response and primes the body to recognize and defend itself against any future re-introduction of cocaine molecules. Inoculation in this way brings about the creation of $\operatorname{IgG}$ antibodies produced against cocaine that can quickly disrupt cocaine as it enters the blood circulation system (Figure 1).

Previous studies utilizing this kind of cocaine immunization have shown promising results in volunteers (humans). The beginning studies were exceptionally encouraging and demonstrated that subjects who had a decent immunizer reaction to the antibody (called TA-CD) essentially diminished their cocaine use [3].

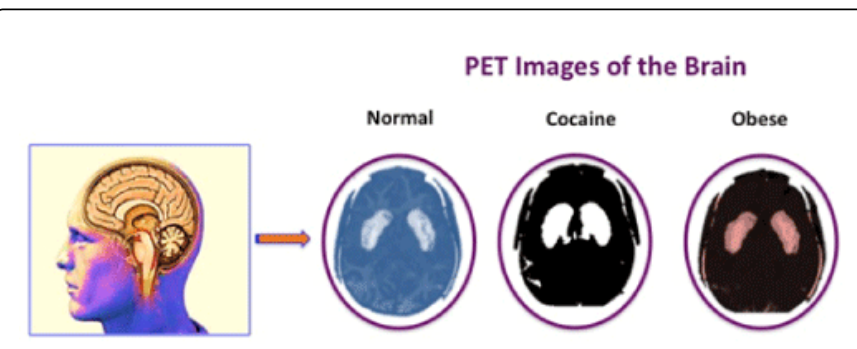

Figure 1: The difference between the brain behaviour with respect to Normal, cocaine addicted, and obese analysis of PET Images.

These early results prompted a larger study that unfortunately did not demonstrate the same achievement [4]. In Kosten's study, three hundred patients struggling with cocaine addiction received either cocaine immunization or placebo injection. The subjects were then stratified into three groups depending on the levels of anti-cocaine IgG resulting from immunization: the individuals who had high IgG titers, the individuals who had low IgG titers, and the placebo-treated individuals with no IgG titers. The results showed no significant differences in cocaine abuse between these groups after 16 weeks. In fact, there were several cases in which the high IgG titer group had higher urine levels of cocaine than those in the low IgG titer group (Figure 2).

Why might a percentage of the subjects who had the best reaction to the immunization have unchanged or even enhanced cocaine dependence? It's imaginable that they were beating the impact of the immunization (i.e. diminishing pleasurable impact of cocaine) by taking more cocaine. This has appeared in a past study where cocaine addicts who received immunization went on to use higher amounts of cocaine than untreated. This can happen in light of the fact that the IgG levels produced by the immunization are limited. Taking higher dosages of cocaine can overwhelm the antibodies so that free cocaine is then accessible to enter the cerebrum. 
Page 2 of 2

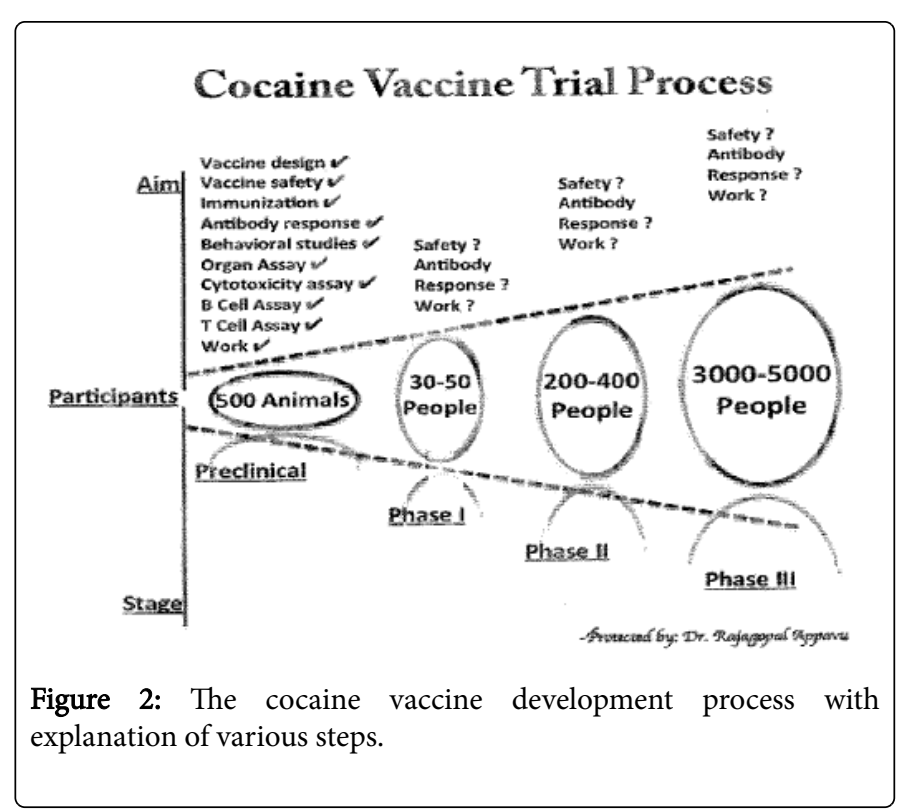

A comparative immunization was made for nicotine reliance (nicVAX), which utilized a pseudomonas protein rather than cholera protein to produce a safe reaction. In any case, tests utilizing this immunization demonstrated comparable findings: promising early studies leading to disappointing Phase 3 studies showing that the antibody was not effective at reducing nicotine dependence.

\section{Conclusions}

These outcomes raise a vital issue. This history of anti-cocaine and anti-nicotine vaccines demonstrates that no immunization can address the entire fundamental neurobiology of habit and substance abuse. For these immunizations to be successful, the patient must be willing to undergo existing mechanisms of treatment including rehabilitation and behavioural counselling. For those patients who desire to free themselves of substance abuse and have access to existing modes of treatment, anti-substance abuse vaccines may be of substantial benefit. Thus, there remains a need to further develop anti-substance abuse vaccines and treatments for these patients. To this end, there is work at present being done to 1) create immunizations that might be more viable than the TA-CD and 2) create compounds that would quickly metabolize the cocaine to inert metabolites.

\section{References}

1. Rajagopal A, Aravinda S, Raghothama S, Shamala N, Balaram P (2012) Aromatic interactions in model peptide $\beta$-hairpins: Ring current effects on proton chemical shifts. Biopolymers 98:185-194.

2. Rajagoapl A, Charles BC, Alexey YK, Joshua DS, Frederick JK, et al. (2015) Enhancing the Magnitude of Antibody Responses through Biomaterial Stereochemistry. ACS Biomater Sci Eng 1: 601-609.

3. Martell BA, Orson FM, Poling J, Mitchell E, Rossen RD, et al. (2009) Cocaine Vaccine for the Treatment of Cocaine Dependence in Methadone Maintained Patients: A Randomized Double-Blind PlaceboControlled Efficacy Trial. Arch Gen Psychiatry 66: 1116-1123.

4. Kosten TR, Domingo CB, Shorter D, Orson F, Green C, et al. (2014)Vaccine for cocaine dependence: A randomized double-blind placebo-controlled efficacy trial. Drug Alcohol Depend 140: 42-47. 